\title{
Clinical, Dermoscopic and In-Vivo Reflectance Confocal Microscopy Evaluation of a Case of Graham Little-Piccardi-Lassueur Syndrome Successfully Treated with Narrowband-UVB Phototherapy
}

\author{
Mariachiara Arisi (D) - Alessandra Gelmetti · Arianna Zanca • \\ Mariateresa Rossi · Luisa Lorenzi · Paolo Incardona · Piergiacomo Calzavara-Pinton • \\ Marina Venturini
}

Received: May 6, 2020 / Published online: June 12, 2020

(C) The Author(s) 2020

\section{ABSTRACT}

Graham Little-Piccardi-Lassueur syndrome (GLPLS) is a rare variant of lichen planopilaris, characterized by a triad of clinical signs including follicular spinous papules on the body area, scarring alopecia of the scalp and non-scarring alopecia of the groin and/or axilla. To date, fewer than 50 cases have been described in the literature. We first report a case of GLPLS investigated with non-invasive techniques such as dermoscopy and in vivo reflectance confocal microscopy (RCM) and successfully treated with narrowband-UVB (NBUVB) phototherapy.

Keywords: Cicatricial alopecia; Graham LittlePiccardi-Lassueur syndrome; Lichen spinulosus; NB-UVB phototherapy

Digital Features To view digital features for this article go to https://doi.org/10.6084/m9.figshare.12416405.

M. Arisi $(\bowtie) \cdot$ A. Gelmetti · A. Zanca · M. Rossi ·

P. Calzavara-Pinton · M. Venturini

Department of Dermatology, University of Brescia, ASST Spedali Civili of Brescia, Brescia, Italy

e-mail: mariachiara.arisi@gmail.com

L. Lorenzi · P. Incardona

Pathology Department, University of Brescia, ASST

Spedali Civili di Brescia, Brescia, Italy

\section{Key Summary Points}

Graham Little-Piccardi-Lassueur syndrome (GLPLS) is a rare variant of lichen planopilaris.

The disease has never been treated with narrowband UVB (NB-UVB) or investigated by in vivo confocal microscopy.

We aimed to report clinical and noninvasive evaluation of NB-UVB efficacy in a patient affected by GLPLS.

\section{INTRODUCTION}

Graham Little-Piccardi-Lassueur syndrome (GLPLS) is a rare variant of lichen planopilaris, characterized by a triad of clinical signs including follicular spinous papules on the body area, scarring alopecia of the scalp and non-scarring alopecia of the groin and/or axilla $[1,2]$.

Here we report the case of a 45-year-old female affected by this rare skin condition, treated with narrowband-UVB (NB-UVB) phototherapy. In this case, dermoscopy and in vivo reflectance confocal microscopy (RCM) were performed to evaluate response to treatment, 
supplementing clinical evaluation and avoiding confirmatory biopsies. The aim of the present case report was to highlight NB-UVB efficacy as a new treatment option for GLPLS, which was confirmed by advanced non-invasive diagnostic techniques. This case report was conducted according to the principles of good clinical practice guidelines ICH GCP and according to the ethical principles of the Declaration of Helsinki. The patient provided her written informed consent for the publication of her case.

\section{CASE REPORT}

A 45-year-old female presented with a 13-year history of papular spiny eruptions on the trunk and limbs. She reported severe pruritus and relevant concern about her skin condition.

The patient was otherwise in good health. Family history was positive for psoriasis in the sister. Treatment history included topical steroids and oral antihistamines, without relief.

At first clinical examination, she presented hyperkeratotic follicular papules, particularly affecting the upper and lower limbs (Fig. 1a, g). Furthermore, an apparently scarring type of alopecia of the scalp and an apparently nonscarring type of alopecia of the pubis region and axillae were noted (Fig. 1c, e). A pull test could not be performed on these sites because of the inconsistent number of hairs.

Papular lesions on the body presented before the alopecia of the scalp and pubis, while the axillae have been involved for 6 years.

Dermoscopic evaluation of different areas was performed (Vivascope 1500, Lucid Technologies, Henrietta, NY). On the affected skin of the shoulder and thigh, it revealed multiple round-to-oval yellowish areas with keratotic follicular plugs, surrounded by mild erythema (Fig. 1b, h). Dermoscopy of the scalp showed blue-gray dots in a target pattern surrounding hair follicles, perifollicular scaling and tufted hair (Fig. 1d). These features characterize a scarring type of alopecia. Corresponding to the groin and pubis, yellow dots and isolated exclamation mark hair were detected as findings of non-active alopecia areata (Fig. 1f).

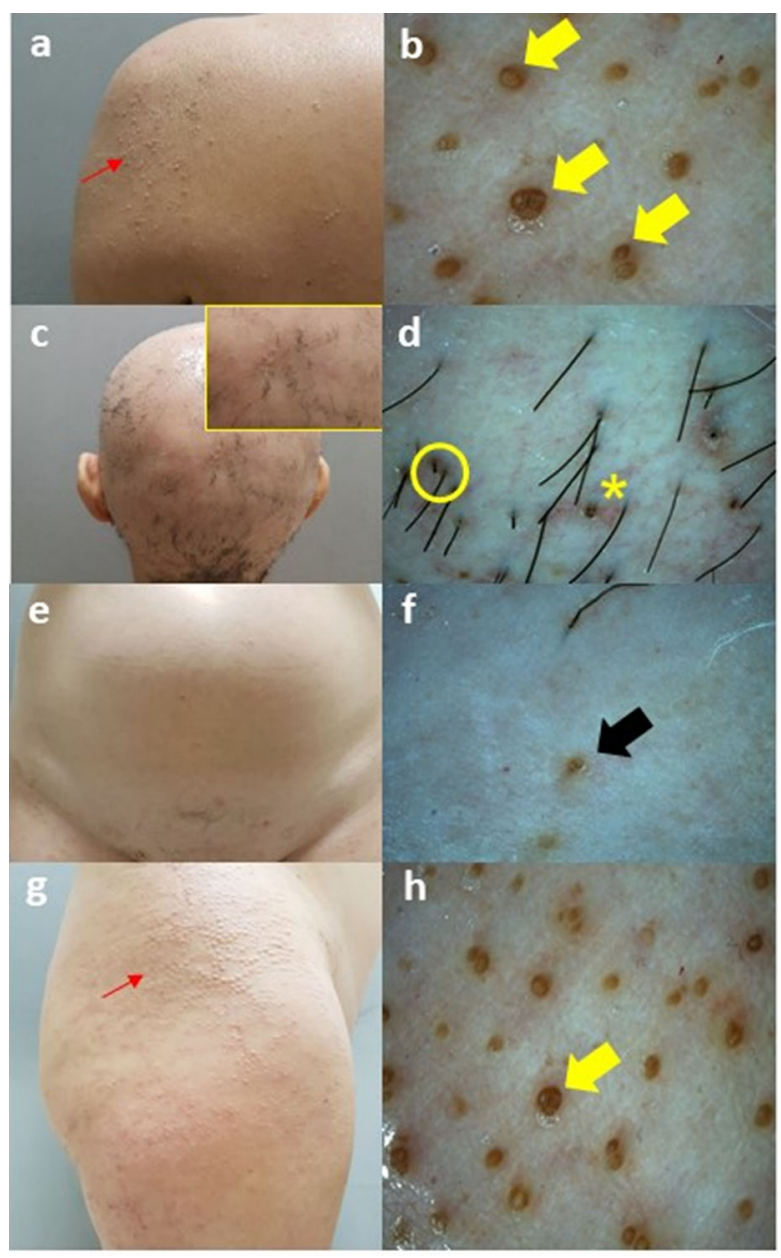

Fig. 1 Baseline clinical and dermoscopic evaluation: a Spiny papules of the left shoulder (red arrow); b dermoscopic pattern $(20 \times)$ of affected skin of the shoulder: multiple round-to-oval yellowish areas with keratotic follicular plugs surrounded by mild erythema (yellow arrows); c scarring alopecia of the scalp and detail (inset); $\mathbf{d}$ dermoscopic pattern of scarring alopecia of the scalp $(20 \times)$ : blue-gray dots in a target pattern, tufted hair (yellow circle) and perifollicular scaling (asterisk); e clinical picture of non-scarring alopecia of pubis; e dermoscopic pattern $(20 \times)$ showing isolated yellow dots (black arrow); g spiny papules of the right thigh (red arrow); $\mathbf{h}$ dermoscopic pattern $(20 \times)$ of affected skin of the thigh: multiple round-to-oval yellowish areas with keratotic follicular plugs surrounded by mild erythema (yellow arrows)

In vivo RCM evaluation of the lesions on the shoulder was also performed (Vivascope 1500, Lucid Technologies, Henrietta, NY) showing hyperreflctive multiple round-to-oval areas with 
keratotic follicular plugs and hyperkeratosis (Fig. $2 \mathrm{a}-\mathrm{c}$ ) and loss of papillae cyclicity as an indirect sign of the presence of junctional inflammatory infiltrate.

A 5-mm punch biopsy of a papular lesion of the left shoulder was performed. Histologic examination revealed dilated follicular ostia with compact hyperkeratosis, focally associated with a perifollicular and perivascular lichenoid inflammatory infiltrate composed of little nonatypical lymphocytes. Junctional vasculopathy and rare acidophilic bodies were also detected. Periodic acid Schiff (PAS) staining was negative for mycotic hyphae and spores (Fig. 2d-f).

All these features were compatible with the diagnosis of lichen spinulosus.

A further 5-mm punch biopsy of the scalp was performed revealing diffuse fibrotic tissue, mild perivascular and perifollicular mononuclear inflammatory infiltrate and the presence of arrector pili muscles without associated follicles. These findings confirmed the clinical suspicion of scarring alopecia of the scalp.

Based on the above clinical and instrumental findings, diagnosis of GLPLS was made, and the patient was started on treatment with NB-UVB phototherapy (Medisun 311, Schulze \& Böhm $\mathrm{GmbH}$, Brühl, Germany) twice weekly. Irradiance was measured with a handle radiometer (Waldmann Variocontrol, Herbert Waldmann Gmbh \& Co., 78,056 Villingen-Schwenningen, Germany) and found to be $5.33 \mathrm{~mW} / \mathrm{cm}^{2}$ at skin level. During the treatment period the patient was allowed to apply only glycerin emollient cream.

After 33 irradiations (medium dose $0.52 \mathrm{~J} /$ $\mathrm{cm}^{2}$ ) an objective improvement of skin lesions

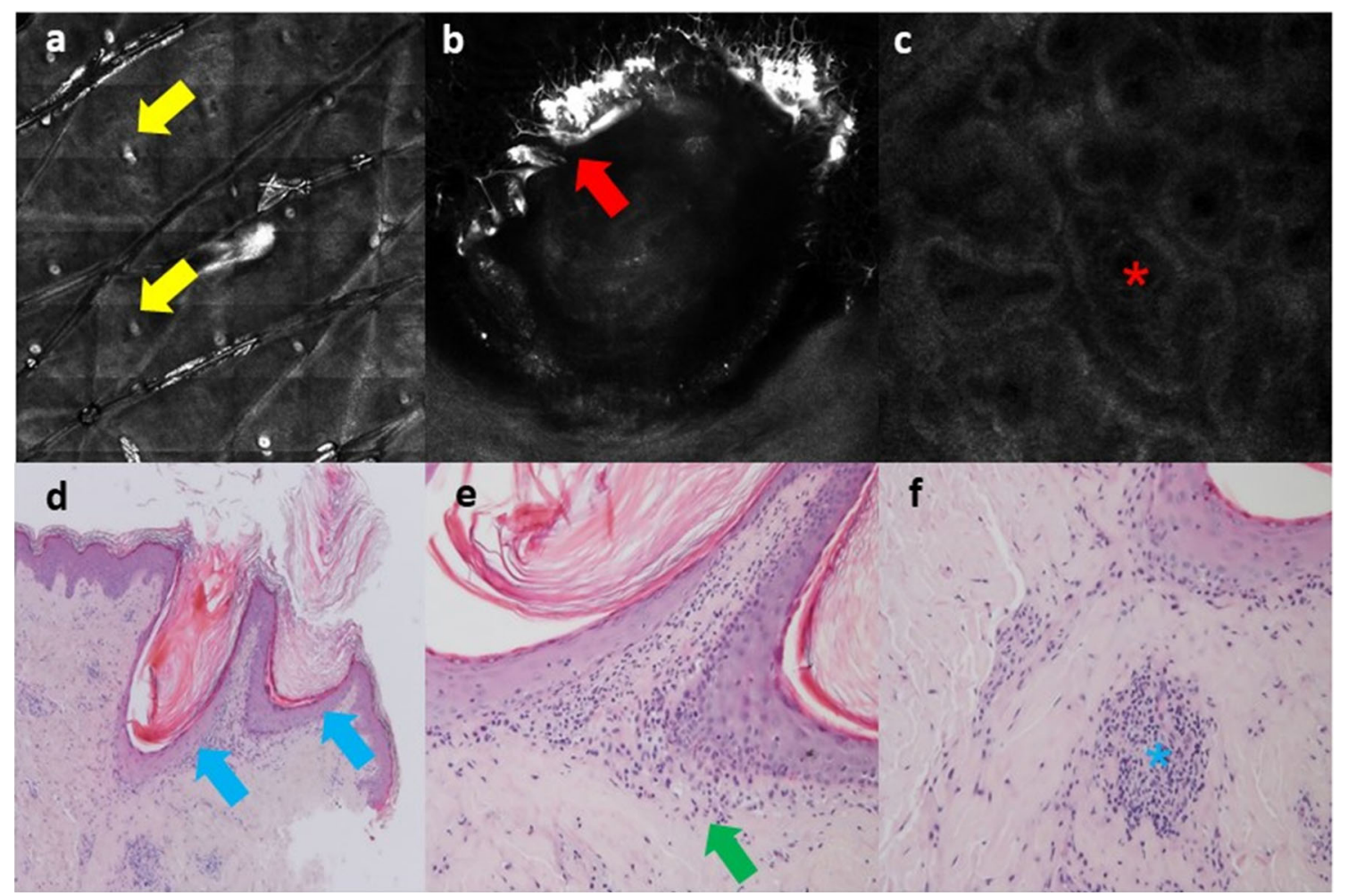

Fig. 2 In vivo RCM of a papular lesion of the shoulder: a multiple round-to-oval areas with keratotic follicular plugs (yellow arrows); b follicular hyperkeratosis (red arrow); c perivascular infiltrate (red asterisk showing a vessel). Histologic analysis of the same lesion $(E / E \mathbf{o})$ : d dilated ostia with hyperkeratosis (10x; blue arrows); e lymphocytic inflammatory perifollicular infiltrate $(20 \times$; green arrow); $\mathbf{f}$ detail of perivascular inflammatory infiltrate $(20 \times$; blue asterisk) 
was seen (Fig. 3a, b), confirmed by non-invasive techniques performed at the same body location as baseline. Dermoscopy showed a reduction of the number of keratotic plugs (Fig. 3c) confirmed by in vivo RCM, which also documented the reduction of the perivascular infiltrate (Fig. 3d).

Effects of NB-UVB maintained stable over time: the disease was unchanged at the 3-month follow-up.

\section{DISCUSSION}

GLPLS is a rare type of lichen planopilaris, clinically characterized by the triad of follicular lichen planus of the body, scarring alopecia of the scalp and non-scarring alopecia of the groin and/or axillae. These findings may not present simultaneously.

The syndrome was first described in 1914 by Piccardi and later in 1915 by Graham Little [1]. It presents in adults between 30 and 70 years old and is four times more frequent in females than in males [2].

$\mathrm{T}$ cell-mediated immunity seems to be involved in the pathogenesis, since GLPLS is considered one of the three variants of lichen planopilaris [3], but the inflammatory response trigger remains unclear. Immunologic factors such as the presence of HLA-DR may be involved: a case of a familial pattern correlated with the presence of HLA-DR1 in a mother and a daughter both affected by the syndrome has been reported [4].
Recently, the presence of autoantibodies to centromere passenger INCENP has been documented [5]. This protein is a major component of the centromere during several phases of the mitotic cell cycle, where it plays an essential role in chromosome segregation and participates in the mechanisms regulating mitosis [5].

More than one case report documented the possible association of the syndrome with hormonal imbalances, in particular androgen insensitivity syndrome and pituitary insufficiency [6].

A more recent hypothesis is the antigen mimicry mechanism, which suggests the possible role of exogen antigens, such as infective agents, in triggering the lymphocytic inflammatory response characteristic of the syndrome [7].

Our patient presented with all the typical clinical signs of GLPLS, and no apparent cause was found. Clinical examination and anamnestic data allowed us to make the diagnosis, which was confirmed by histology.

To our knowledge, this is the first report of GLPLS described with non-invasive techniques such as dermoscopy and in vivo RCM. To date, only lichen planus has been studied by RCM, describing spongiosis, hypergranulosis, necrotic keratinocytes, focal or diffuse inflammatory infiltrate, dilated blood vessels and interface dermatitis as common features [8].

In our case, we noticed as main RCM diagnostic features the presence of hyperreflecting structures representing hyperkeratosis and loss of papillae cyclicity as an indirect sign of the presence of an inflammatory infiltrate.

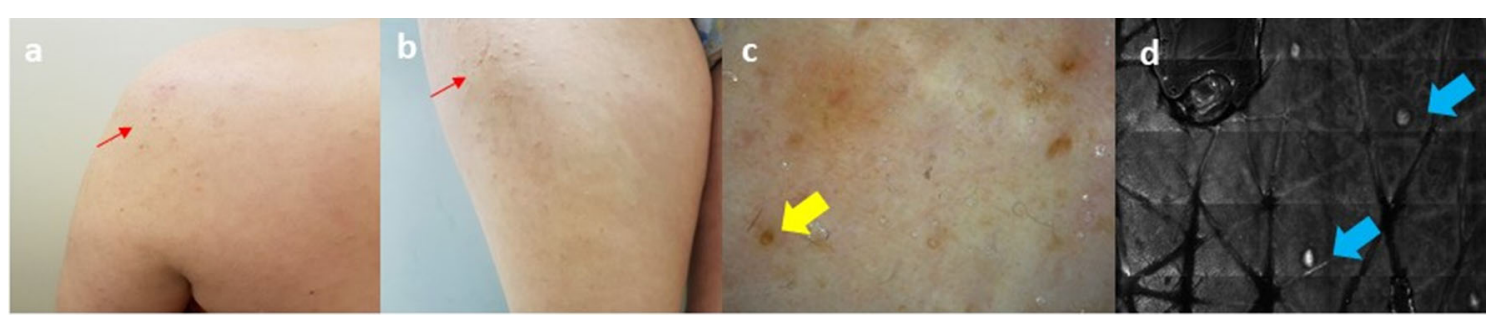

Fig. 3 Clinical and non-invasive evaluation of skin lesions after NB-UVB: a clinical reduction of papular skin lesions of the left shoulder and $\mathbf{b}$ the right thigh (red arrows); c dermoscopy of the shoulder $(20 \times)$ : reduction of the number of keratotic plugs (yellow arrow); $\mathbf{d}$ reduction of the number of follicular keratotic plugs at in vivo RCM evaluation (shoulder, blue arrows) 
Several treatment strategies have been proposed in classic lichen spinulosus, although no gold standard therapy has been recognized so far [9]. In single case reports several drugs have been proposed for the treatment of cutaneous lesions: the most used are topical, systemic and intralesional corticosteroids. Few patients were treated with cyclosporine and hydroxychloroquine. Retinoids and photochemotherapy (PUVA) were also used. All these treatments resulted in a complete or partial response of papular skin lesions and stabilization of the alopecia.

Considering the inflammatory nature of lichen spinulosus and the patient's refusal to undergo any systemic treatment, we decided to treat her with NB-UVB phototherapy, according to its well-known immunomodulatory effect [10].

After 33 irradiations, the overall appearance of the skin clearly improved with a reduction of spiny papular lesions (Fig. 3a, b), which became much softer and less rough to touch.

No improvement of the scalp or pubis alopecia was seen.

Dermoscopy performed on the shoulder after treatment documented a reduction of the number of keratotic plugs (Fig. 3c). This finding was also confirmed by in vivo RCM, which highlighted the reduction of the perivascular infiltrate (Fig. 3d).

\section{CONCLUSIONS}

We performed, for the first time, dermoscopy and in vivo RCM evaluation in a patient affected by GLPLS treated with NB-UVB. These noninvasive techniques supported the clinical and histologic diagnosis and objectively documented the improvement of the patient's skin condition due to the therapy without useless and repetitive confirming biopsies. To date, use of NB-UVB in GLPLS has never been described. Our experience, although limited to a single case, first supported NB-UVB's effectiveness in this rare skin disease.

\section{ACKNOWLEDGEMENTS}

The authors thank the participants of the study with special appreciation to the nursing staff of the phototherapy unit.

Funding. No funding or sponsorship was received for this study or publication of this article.

Authorship. All named authors meet the International Committee od Medical Journal Editors (ICMJE) criteria for authorship for this article, take responsibility for the integrity of the work as a whole, and have given their approval for this version to be published.

Disclosures. Mariachiara Arisi, Alessandra Gelmetti, Arianna Zanca, Mariateresa Rossi, Luisa Lorenzi, Paolo Incardona, Marina Venturini. Piergiacomo Calzavara-Pinton is a member of the journal's Editorial Board.

Compliance with Ethics Guidelines. This case report was conducted according to the principles of good clinical practice guidelines ICH GCP and according to the ethical principles of the Declaration of Helsinki. The patient provided her written informed consent for the publication of her case.

Open Access. This article is licensed under a Creative Commons Attribution-NonCommercial 4.0 International License, which permits any non-commercial use, sharing, adaptation, distribution and reproduction in any medium or format, as long as you give appropriate credit to the original author(s) and the source, provide a link to the Creative Commons licence, and indicate if changes were made. The images or other third party material in this article are included in the article's Creative Commons licence, unless indicated otherwise in a credit line to the material. If material is not included in the article's Creative Commons licence and your intended use is not permitted by statutory regulation or exceeds the permitted use, you will need to obtain permission directly from the copyright holder. To view a copy of this licence, 
visit http://creativecommons.org/licenses/by$\mathrm{nc} / 4.0 /$.

\section{REFERENCES}

1. Graham-Little EG. Folliculitis decalvans et atrophicans. Br J Dermatol. 1915;27:183-5.

2. Odom RB, James WD, Berger TG. Lichen planus and related conditions. In: Odom RB, James WD, Berger TG, editors. Andrews' Diseases of the skin. 9th ed. Philadelphia: WB Saunders Company; 2000. p. 274-275.

3. Assouly P, Reygagne P. Lichen planopilaris: update on diagnosis and treatment. Semin Cutan Med Surg. 2009;28:3-10.

4. Viglizzo G, Verrini A, Rongioletti F. Familial Lassueur-Graham-Little-Piccardi syndrome. Dermatology. 2004;208:142-4.

5. Rodrìguez-Bayona $\mathrm{B}$, Ruchaud $\mathrm{S}$, Rodrìguez $\mathrm{C}$, et al. Autoantibodies against the chromosomal passenger protein INCENP found in a patient with Graham Little-Piccardi-Lassueur syndrome. J Autoimmune Dis. $2007 ; 4: 1$.
6. Vega Gutìerrez J, Miranda-Romero A, Pèrez MF, Martìnez GG. Graham Little-Piccardi-Lassueur syndrome associated with androgen insensitivity syndrome (testicular feminization). J Eur Acad Dermatol Venereol. 2004;18:463-6.

7. Tchernev G, Nenoff P. Antigen mimicry followed by epitope spreding: A pathogenetic trigger for the clinical morphology of lichen planus and its transition to Graham Lassueur Piccardi Little Syndrome and keratosis lichenoides chronica- Medical hypotheses or reality? An Bras Dermatol. 2009;84: 682-8.

8. Ianosi SL, Forsea AM, Lupu M, et al. Role of modern imaging techniques for the in vivo diagnosis of lichen planus. Exp ther med. 2018;17:1052-60.

9. Errichetti E, Figini M, Croatto M, Stinco G. Therapeutic management of classic lichen planopilaris: a systematic review. Clin Cosm Inv Dermatol. 2018;11:91-102.

10. Sigmundsdottir $\mathrm{H}$, Johnston A, Gudjonsson JE, Valdimarsson H. Narrowband-UVB irradiation decreases the production of pro-inflammatory cytokines by stimulated T cells. Arch Dermatol Res. 2005;297:39-42. 